\title{
Intensely enhanced Fallopian canal as a surrogate marker for vasculitis in the ear: Our experience in four patients
}

\author{
Taro FUJIKAWA ${ }^{1}$, Keiji Honda ${ }^{1}$, Taku Ito ${ }^{1}$, Mitsuhiro Kishino ${ }^{1}$, Naoki Kimura ${ }^{1}$, Natsuka \\ Umezawa $^{1}$, Mana Hirano ${ }^{2}$, Natsuki Aoki ${ }^{1}$, Yoshiyuki Kawashima ${ }^{1}$, and Takeshi Tsutsumi ${ }^{1}$ \\ ${ }^{1}$ Tokyo Medical and Dental University \\ ${ }^{2}$ National Defense Medical College
}

June 15,2020

\begin{abstract}
1. Granulomatosis with polyangiitis (GPA) and eosinophilic granulomatosis with polyangiitis (EGPA) frequently affect the ear, resulting in the development of external otitis, otitis media, sensorineural hearing loss, and facial paresis. 2. The lack of specific methods for detecting vasculitis of the ear occasionally hinders the early diagnosis of otologic involvement of GPA and EGPA. 3. Contrast-enhanced, 3D T1-weighted magnetic resonance imaging with gradient-echo sequences (CE-3D-GRE) demonstrate intense enhancement in the tympanic and mastoid segments of the Fallopian canal in spite of the absence of facial paresis. 4. Maximum intensity projection images clearly display an enhanced Fallopian canal, which was associated with adjacent inflammatory lesions. 5. Intense enhancement of the Fallopian canal on CE-3D-GRE can be used as a surrogate marker of otologic involvement of GPA and EGPA, which may enable early diagnosis, monitoring, and therapy optimisation in a less invasive manner.
\end{abstract}

Title: Intensely enhanced Fallopian canal as a surrogate marker for vasculitis in the ear: Our experience in four patients

\section{KEY POINTS}

1. Granulomatosis with polyangiitis (GPA) and eosinophilic granulomatosis with polyangiitis (EGPA) frequently affect the ear, resulting in the development of external otitis, otitis media, sensorineural hearing loss, and facial paresis.

2. The lack of specific methods fordetectingvasculitis of the ear occasionally hinders the early diagnosis of otologic involvementof GPA and EGPA.

3. Contrast-enhanced, 3D T1-weighted magnetic resonance imaging with gradient-echo sequences (CE3D-GRE) demonstrate intense enhancement in the tympanic and mastoid segments of the Fallopian canalin spite of the absence of facial paresis.

4. Maximum intensity projection imagesclearly display an enhanced Fallopian canal,which was associated with adjacent inflammatory lesions.

5. Intense enhancement of the Fallopian canal on CE-3D-GRE can be used as a surrogate marker of otologic involvement of GPA and EGPA, which may enable early diagnosis, monitoring, and therapy optimisation in a less invasive manner.

\section{INTRODUCTION}

Antineutrophil cytoplasmic antibody (ANCA)-associated vasculitis (AAV) is a necrotising vasculitis that predominantly affects small-size vessels ofany organ. Among AAV, granulomatosis with polyangiitis (GPA) and eosinophilic granulomatosis with polyangiitis (EGPA) frequently affect the eartodevelopvarious otologic manifestations, including external otitis, otitis media, sensorineural hearing loss (SNHL), and facial 
paresis. ${ }^{1,2}$ However, the lack of specific imaging methods ${ }^{1}$ occasionally hinders early diagnosis of otologic involvement, which is essential for preventing irreversible hearing loss and other long-term complications caused by systemic AAV.

Temporal bone study of GPA shows evidence of vasculitis in the Fallopian canal with and without the clinical presentation of facial paresis. ${ }^{3}$ The stylomastoid artery(SMA) in the Fallopian canalsupplies the posterior half of the external ear canal, tympanic cavity, mastoid, and facial nerve, ${ }^{4}$ which are consistent with frequent sites of otologic involvement in GPA and EGPA. In the present study, using contrast-enhanced, 3D T1weighted,magnetic resonance imaging (MRI) with gradient-echo sequences (CE-3D-GRE), ${ }^{5,6}$ we tested the hypothesis that this imaging protocol could detect signs of vasculitis in the Fallopian canal, which may cause otologic involvement in AAV patients.

\section{MATERIALS AND METHOD}

\section{Subjects}

We prospectively performed CE-3D-GRE at the onset or at relapse of the disease, and during remission on four AAV patients who presented with otologic manifestations as major initial symptoms. Diagnosis for GPA was made based on the European Medicines Agency (EMEA) vasculitis classification algorithm of 2007 because it requires characteristic symptoms of the respiratory tract suggestive of GPA, including otologic involvement, and positive serology for ANCA, and does not rely on histological confirmation for diagnosis. Diagnosis of EGPA was made in accordance with the 1990 American College of Rheumatology (ACR) criteria.

\section{Scanning protocol of CE-3D-GRE}

A dedicated 8-channel head coil was used on a 3.0 Tesla MRI scanner, with the 3D fast-spoiled gradient-echo sequence (SPGR) (SignaHDxt; GE Healthcare, Waukesha, WI, USA) or with the 3D fast-field echo sequence (FFE) (Ingenia CX; Philips Healthcare, Best, the Netherlands). The parameters for SPGR were as follows: $\mathrm{TR} / \mathrm{TE}=7.4 / 2.8 \mathrm{~ms}$; flip angle, $20^{\circ}$; field of view, $20 \times 20 \mathrm{~cm}$; matrix size, $288 \times 256$; section thickness, $1.5 \mathrm{~mm}$; slice spacing, $1.5 \mathrm{~mm}$; and acquisition time, 110seconds. The parameters for FFE were as follows: $\mathrm{TR} / \mathrm{TE}=16.0 / 4.6 \mathrm{~ms}$; flip angle, $20^{\mathrm{O}}$; field of view, $22 \times 22 \mathrm{~cm}$; matrix size, $448 \times 448$; section thickness, $1.0 \mathrm{~mm}$; slice spacing, $0.5 \mathrm{~mm}$; and acquisition time, 218 seconds. Contrast-enhanced images were acquired immediately after an intravenous bolus injection of $0.1 \mathrm{ml} / \mathrm{kg}$ gadobutrol (Gadovist 1.0; BayerSchering Pharma, Berlin, Germany) or $0.2 \mathrm{ml} / \mathrm{kg}$ megluminegadoterate (Magnescope; Guerbet,Tokyo, Japan).

\section{Signal intensity assessment of the facial nerve}

Multi-planner reconstruction (MPR) and maximum intensity projection (MIP) images were obtained using RadiAnt DICOM Viewer (Medixant, Poznan, Poland). For five affected ears, anatomical segments of the facial nerve were identified on MPR images, and the signal intensity of each segment was assigned a value by the first author as follows: 0 , no signal; 1 , faint visualisation; 2 , signal equivalent to normal cerebellum; 3 , signal equivalent to enhancing dural sinus. ${ }^{6}$ Furthermore, we compared the signal intensity of the mastoid segment between before and after remission induction in three affected ears. Four otolaryngologists, two rheumatologists, and one radiologist were blinded to the clinical information and scored each individual case independently in a single session. For comparison, Case 4 was excluded due to being under treatment.

\section{RESULTS}

Three GPA and one EGPA were included in this study (Table 1). Case 1 and Case 2had recurrenceof acute SNHL with external otitis of the posterior canal wall during an attempted taper of prednisolone (Fig. S1). Case 3 and Case 4 presented with persistent otitis media with aseptic discharge and gradual progressive SNHL as an initial symptom (Fig. S1). Bilateral ears were affected at the initial onset in Case 4. Vertigo with a complete canal paresis was seen only in Case 3.Simultaneous facial paresis was absent in all patients.

The signal intensity of each segment of the facial nerve was assessed in five affected ears (Table 2). The geniculate ganglion was normally enhanced with a moderate to intense signal intensity withoutan obvious 
difference between affected and unaffected ears (Fig. 1A). In all affected ears, the temporal and mastoid segments were significantly enhanced(Fig. 1B, 1C, 1D, 2A, 2B). The enhancement was detected along the course of the Fallopian canal on MIP images,and closely associated with inflammation inthe promontory, mastoid, and eustachian tube (Fig. 2C, 2D). After immunosuppressive therapy,the enhancement was attenuated to a level similar to that of unaffected ears (Fig. 1E), with a mean signal intensity score of the mastoid segment from 2.7 at onset to 1.4 inremission. On the other hand, the cisternal, canalicular, and labyrinthine segments were faintly enhanced and hardly detectable(Fig. 1A). Pachymeningitis or other intracranial involvements were not found.

\section{DISCUSSION}

CE-3D-GRE is able to visualise the normal facial nerve in the geniculate ganglion, and the tympanic and mastoid segmentswith high resolutionand moderate signal intensitydue to the presence of the intrinsic plexus. ${ }^{5,6} \mathrm{In}$ pathological conditions, includingRamsay Hunt syndrome and Bell's palsy,the facial nerve is intensely enhanced because disruption of the blood-nerve barrier causes leakage and further accumulation of contrast material inside the Fallopian canal. ${ }^{5}$

We performed CE-3D-GRE at 3.0 Tesla on four patients with otologic manifestations of AAV as the major initial symptoms. In this study, intense enhancement in the tympanic and mastoid segments of the Fallopian canal was clearly associated with development of otologic symptoms, including external otitis, otitis media, and SNHL. The enhancement was attenuated to the normal level in accordance with clinical improvement after immunosuppressive treatment. The results suggestthat intense enhancement of the Fallopian canal reflects disease activity of vasculitis in the ear and can be used as a surrogate marker of otologic involvement of AAV. It is clinically important because the imaging method may enable early diagnosis, monitoring, and therapy optimisation of otologic involvement of AAV in a less invasive manner.

The findings in this study provide further insights into the pathophysiology of vasculitis of the ear. The SMA enters the Fallopian canal and predominantly supplies the mastoid and tympanic segment of the facial nerve, ${ }^{4,7}$ giving off numerous branches to the mastoid, tympanic cavity, and the posterior wall of the external ear canal, which are in line with frequent otologic manifestations in AAV. Our findings on CE-3D-GRE were consistent with pathologic evidence of vasculitis in the mastoid and tympanic segments in patients with $\mathrm{GPA}^{3}$ and polyarteritis nodosa, ${ }^{8}$ which is also known to present with otitis media and facial paresis. We therefore consider that otologic involvement of AAV is at least in part caused by vasculitis of the SMA and imaging shows intense enhancement in the mastoid and tympanic segments of the Fallopian canal on CE-3D-GRE.

Furthermore, our results support vasculitis in the cochlea as anetiology of SNHL, ${ }^{3}$ rather than eight-cranialnerve neuropathy. ${ }^{2} \mathrm{We}$ could not find significant enhancement in the geniculate ganglion or internal auditory canal on CE-3D-GRE(Fig. 1A). One possible mechanism of nerve involvement in AAV is vasculitis originating in arterio-venous anastomoses (AVAs), which are 7 ? $\mathrm{m}$ in a diameter ${ }^{9}$ and exist only in the stria vascularis and spiral ganglion of the cochlea ${ }^{9}$ and the Fallopian canal ${ }^{7}$ in the temporal bone. This hypothesis is able to explain several features of otologic manifestations of AAV. First, microembolism of AVAs in the stria vascularis and spiral ganglion may cause a reduction in the endocochlear potential and reversible SNHL. ${ }^{3}$ Second, vertigo is less frequent and less severe compared to SNHL, ${ }^{2,3}$ due to the lack of AVAs in the periphery of both the macular and crista. ${ }^{10}$ Progression of the vasculitic process may cause arterial embolism and subsequent ischaemic necrosis of the peripheral organs. Lastly, facial paresis is not always in concert with vasculitis in the Fallopian canal, probably due to occasional connections between AVAs and the vasa nervorum of the facial nerve. ${ }^{7}$

This study had limitations due to its study design and the small number of included patients. Despite this, we showed a clear relationship between clinical presentations and radiological findings using 3D MRI acquired at 3.0 Tesla. Further studies are necessary for assessment of the sensitivity and specificity of the imaging protocol using more patients and comparison with other ear diseases.

\section{REFERENCES}


1. Kuhn D, Hospowsky C, Both M, Hey M, Laudien M. Manifestation of granulomatosis with polyangiitis in head and neck. ClinExpRheumatol 2018;36:78-84.

2. Seccia V, Fortunato S, Cristofani-Mencacci L, Dallan I, Casani AP, Latorre M, et al. Focus on audiologic impairment in eosinophilic granulomatosis with polyangiitis. Laryngoscope 2016;126:2792-2797.

3. Santos F, Salviz M, Domond H, Nadol JB. Otopathology of Vasculitis in Granulomatosis WithPolyangitis. OtolNeurotol 2015;36:1657-1662.

4. Nager GT, Nager M. The arteries of the human middle ear, with particular regard to the blood supply of the auditory ossicles. Ann OtolRhinolLaryngol 1953;62:923-949.

5. Hong HS, Yi BH, Cha JG, Park SJ, Kim DH, Lee HK, et al. Enhancement pattern of the normal facial nerve at 3.0 T temporal MRI. Br JRadiol 2010;83:118-121.

6. Dehkharghani S, Lubarsky M, Aiken AH, Kang J, Hudgins PA, Saindane AM. Redefining normal facial nerve enhancement: healthy subject comparison of typical enhancement patterns-unenhanced and contrast-enhanced spin-echo versus 3D inversion recovery-prepared fast spoiled gradient-echo imaging. Am JRoentgenol 2014;202:1108-1113.

7. Bosatra A. Some observations on the vascularization of the VIIth nerve. JLaryngolOtol 1956;70:605-613.

8. Joglekar S, Deroee AF, Morita N, Cureoglu S, Schachern PA, Paparella M. Polyarteritis nodosa: a human temporal bone study. Am JOtolaryngol 2010;31:221-225.

9. Axelsson A. The vascular anatomy of the cochlea in the guinea pig and in man. ActaOtolaryngol. 1968; $3+$.

10. Mazzoni A. The vascular anatomy of the vestibular labyrinth in man. ActaOtolaryngol Suppl. 1990;472:1-83.

\section{FIGURE LEGENDS}

Fig. 1. Imaging of the Fallopian canal (arrowheads) in Case 3 at onset (A - D, fat-saturated SPGR) and during remission (E, fat-suppressed FFE). (A) The cisternal and canalicular segments, and geniculate ganglions (g) in an axial section. (B) The tympanic segments in an axial section. (C - E) The mastoid segments and the eustachian tube (arrow) in an axial section (C) and coronal sections (D, E). Intense enhancement in the tympanic and mastoid segments at onset is attenuated in remission. Asterisks indicate the petrous apices with fatty marrow collection.

Fig. 2. Imaging of the Fallopian canal (arrowheads) in Case 4 at onset on fat-suppressed SPGR. (A) The tympanic segments in an axial section. (B) The mastoid segments in a coronal section. (C, D) Maximum intensity projection images in the range of dashed lines in A.Inflammatory changes in the promontory, mastoid, and eustachian tube (arrows) are closely associated with intensely enhanced Fallopian canal.

Fig. S1. Otologic examinations in Case 1 to Case 4. Audiogram at onset and in remission is coloured in black and grey, respectively. Audiogram in remission is not presented in Case 4 because of being under treatment. 

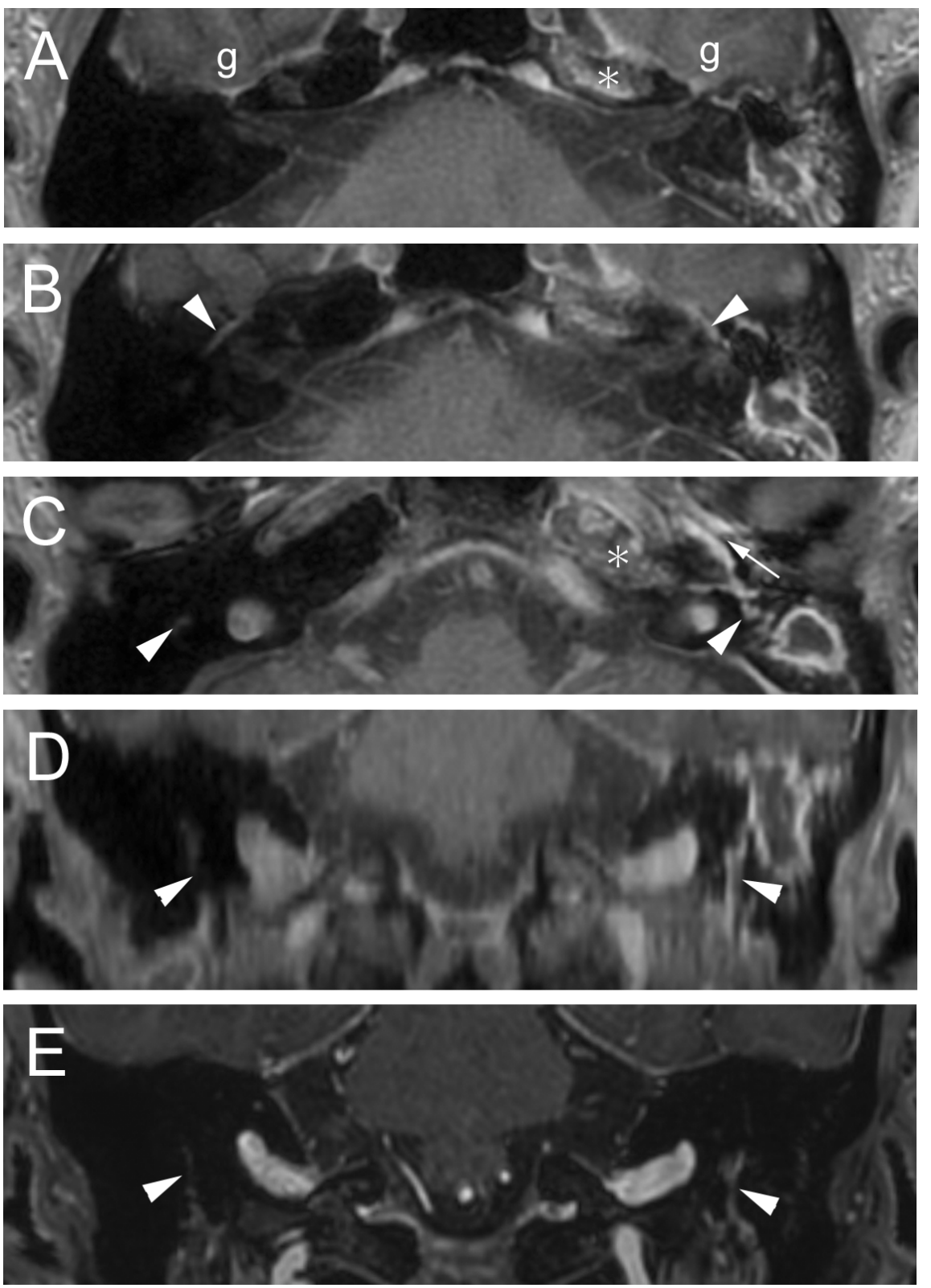

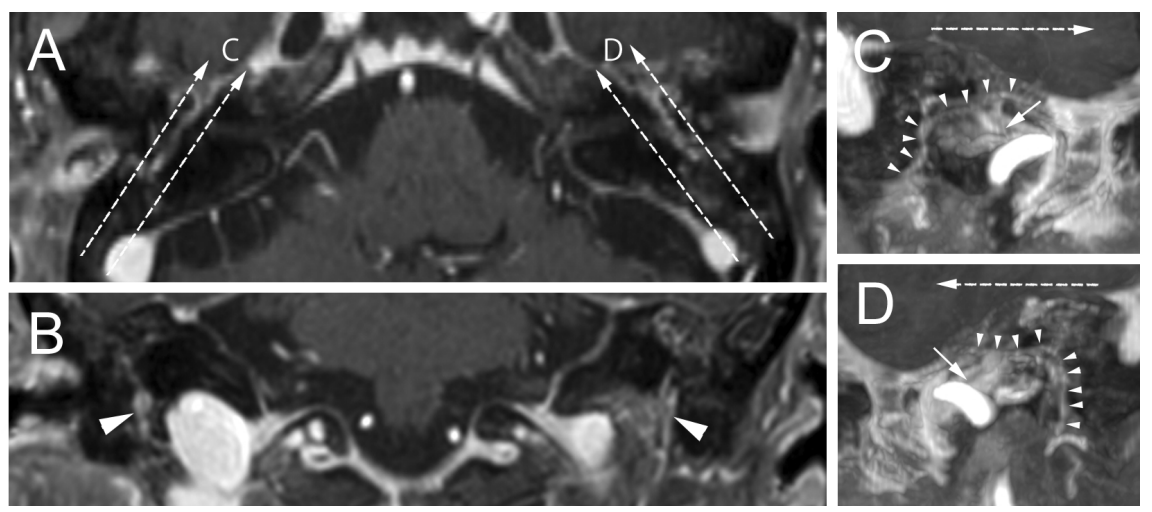

\section{Hosted file}

Table 1.docx available at https://authorea.com/users/333053/articles/459332-intenselyenhanced-fallopian-canal-as-a-surrogate-marker-for-vasculitis-in-the-ear-our-experiencein-four-patients

\section{Hosted file}

Table 2.docx available at https://authorea.com/users/333053/articles/459332-intenselyenhanced-fallopian-canal-as-a-surrogate-marker-for-vasculitis-in-the-ear-our-experiencein-four-patients 